\title{
A Systematic Approach to Prenatal Diagnosis of Transposition of the Great Arteries Using 4-Dimensional Ultrasonography With Spatiotemporal Image Correlation
}

Luís F. Gonçalves, MD, Jimmy Espinoza, MD, Roberto Romero, $M D$, Wesley Lee, MD, Betsy Beyer, MS, Marjorie C. Treadwell, MD, Richard Humes, MD

Abbreviations

STIC, spatiotemporal image correlation; TGA, transposition of the great arteries

Received April 22, 2004, from the Department of Obstetrics and Gynecology, Wayne State University, Detroit, Michigan USA (L.F.G., B.B., M.C.T.); Perinatology Research Branch, National Institute of Child Health and Human Development, National Institutes of Health, Department of Health and Human Services, Bethesda, Maryland USA (L.F.G., J.E., R.R.); Division of Fetal Imaging, William Beaumont Hospital, Royal Oak, Michigan USA (W.L.); and Division of Cardiology, Children's Hospital of Michigan, Wayne State University, Detroit, Michigan USA (R.H.). Revision requested May 24, 2004. Revised manuscript accepted for publication June 1, 2004.

Address correspondence and reprint requests to Roberto Romero, MD, Perinatology Research Branch, National Institute of Child Health and Human Development, Department of Obstetrics and Gynecology, Wayne State University/Hutzel Hospital, 4707 St Antoine Blvd, Detroit MI 48201 USA.

E-mail:warfiela@mail.nih.gov.

Video online at www.jultrasoundmed.org.

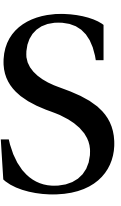

patiotemporal image correlation (STIC) is a recent technological advance in ultrasonographic imaging that allows dynamic multiplanar slicing and surface rendering of the fetal heart. ${ }^{1-5}$ In a previous study, a technique was developed to systematically visualize the outflow tracts from volume data sets acquired with STIC.,4 The addition of color and power Doppler imaging to STIC technology made it possible to dynamically display rendered views of the outflow tracts with minimal manipulation of the volume data set. ${ }^{5}$

Prenatal diagnosis of transposition of the great arteries (TGA) is associated with a significant reduction in both preoperative and postoperative mortality, a decrease in the rate of metabolic acidosis and multiorgan failure during the neonatal period, reduced need for ventilatory support, and shorter hospitalization time. ${ }^{6,7}$ Unfortunately, prenatal detection rates for TGA have been low in most of the studies published to date. ${ }^{8-15}$ Among the reasons for failure to prenatally detect most cases of TGA are the absence of risk factors to identify a target population for screening and the need to systematically examine the outflow tracts to establish the diagnosis. ${ }^{6,16}$ Despite recommendations by scientific societies such as the American Institute of Ultrasound in Medicine to extend the basic fetal cardiac examination to include visualization of the outflow tracts whenever technically feasible, ${ }^{17}$ this examination remains a technical challenge for many sonographers. ${ }^{6,18}$

Four-dimensional volume data set acquisition followed by a systematic approach to image the outflow tracts may reduce the operator dependency of prenatal ultrasonography. Volume acquisition is still operator dependent with current commercially available technology. However, once a good-quality volume data set is acquired, the outflow tracts can be systematically imaged by following algorithms developed for gray scale, ${ }^{3,4}$ color, or power Doppler ${ }^{5}$ imaging. It is anticipated that algorithms developed to image specific cardiac structures with 3- or 4-dimensional volume data sets may eventually become automated by computer software (automated multiplanar imaging). ${ }^{19}$ 
The objective of this report was to describe the ultrasonographic findings of TGA on the basis of a systematic approach of 4-dimensional echocardiography with STIC.

\section{Case Report}

A 43-year-old white patient, gravida 12, para 8038, was approached at $203 / 7$ weeks' gestation to participate in a 4-dimensional ultrasonographic study after a suspected finding of a heart anomaly on 2dimensional ultrasonography. The objective of the examination was to verify whether our previously described techniques for systematic visualization of the outflow tracts using 4-dimensional volume data sets acquired by STIC could be used to examine the heart. ${ }^{3-5}$ The patient gave written informed consent before participation in the study, which was conducted under protocols approved by the Institutional Review Boards of both Wayne State University and the National Institute of Child Health and Human Development.

The obstetric history was remarkable for a previous child with spina bifida, a 20 -week stillbirth of unknown etiology, and 2 first-trimester miscarriages. There was no family history of congenital heart disease and no history of maternal or gestational diabetes or exposure to teratogenic agents known to affect heart development.

Two-dimensional ultrasonography revealed a normal 4-chamber view (Figure 1A and Video 1). Visualization of the outflow tracts showed the great arteries exiting the ventricular chambers in parallel (Figure 1B).

Volume data sets were then acquired by STIC with a Voluson 730 Expert ultrasound system (General Electric Medical Systems, Kretztechnik, Zipf, Austria). Gray scale ultrasonography as well as gray scale plus power Doppler imaging were used during volume acquisition. The acquisition time ranged from 7.5 to 15 seconds, and the acquisition angle ranged from $25^{\circ}$ to $35^{\circ}$. The power Doppler settings at the time of acquisition were as follows: pulse repetition frequency, $5.0 \mathrm{kHz}$; quality status, normal (normal color resolution and medium frame rate); and wall motion filter, midl (the manufacturer preset settings for wall motion filter are low1, low2, mid1, mid2, high1, high2, and max).

A 4-step approach to evaluate the fetal heart using multiplanar display images was applied to a 4-dimensional volume data set obtained with STIC through a transverse sweep of the fetal tho- rax (Figure 2 and Video 2). Images from a normal case at the same gestational age are shown in Figure 3 for comparison (Video 3). Transposition of the great arteries was shown in steps 3 and 4: on rotation of the volume data set around the yaxis (step 3; Figure 2C), 2 vessels could be visualized leaving the ventricular chambers in parallel. Once the reference dot was positioned at the center of the vessel leaving the left ventricle (step 4; Figure 2D, panel A), the sagittal orthogonal view confirmed that this vessel was actually the pulmonary artery (step 4; Figure 2D, panel B).

Volume-rendered power Doppler images of the outflow tracts (Video 4) of a normal case (Figure $4 \mathrm{~A})^{5}$ were compared to this case with TGA (Figure $4 \mathrm{~B})$. In the case with TGA (Figure 4B), the great vessels were visualized leaving the ventricular chambers in parallel, in contrast to crisscrossing of the great arteries in the normal case (Figure $4 \mathrm{~A})$. The technique used to obtain the rendered images is illustrated in Figure 5 (Video 5). ${ }^{5}$

The findings of the 2 previous scans were independently confirmed by fetal and neonatal echocardiography performed at the Department of Fetal Cardiology of the Children's Hospital of Michigan.

\section{Discussion}

Transposition of the great arteries is one of the most common cyanotic congenital heart defects in the neonatal period, ${ }^{20}$ occurring in approximately 0.2 to 0.4 per 1000 live births ${ }^{20}$ and representing $2.5 \%$ to $5 \%$ of all congenital heart defects. ${ }^{21}$ In this anomaly, the pulmonary artery arises from the left ventricle, and the aorta arises from the right ventricle. Associated anomalies may include ventricular septal defects (10\%-20\%), pulmonary stenosis, and coarctation of the aorta. ${ }^{20}$ As the 2 ventricles function in parallel, oxygenation after birth is dependent on mixing of blood through a patent foramen ovale and, to a lesser extent, the ductus arteriosus or a ventricular septal defect.

Prenatal diagnosis of TGA is associated with a reduction in perinatal morbidity ${ }^{7,22}$ and mortality. ${ }^{7}$ Bonnet et $\mathrm{al}^{7}$ compared the preoperative and postoperative morbidity and mortality in 68 neonates with prenatal diagnosis of TGA versus 250 neonates with diagnosis only in the neonatal period. Prenatal diagnosis of TGA was associated with (1) a reduced requirement for mechanical ventilation ( $18 \%$ [ 12 of 68 ] versus $38 \%$ [ 96 of 250 ]; 


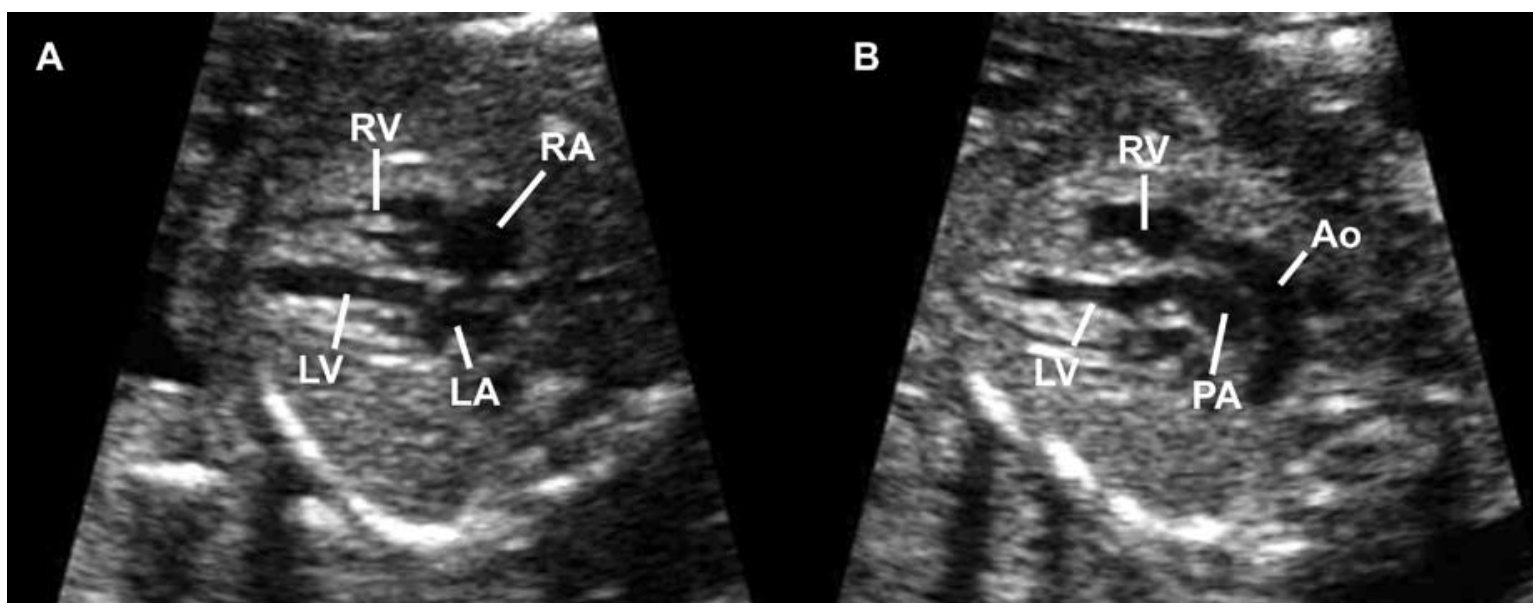

Figure 1. Two-dimensional ultrasonographic images of TGA. A, Normal 4-chamber view of the fetal heart. B, Visualization of the outflow tracts. The great arteries leave the ventricles in parallel: the pulmonary artery is connected to the left ventricle, and the aorta is connected to the right ventricle. Ao indicates aorta; LA, left atrium; LV, left ventricle; PA, pulmonary artery; RA, right atrium; and RV, right ventricle.

Figure 2. Multiplanar slicing of TGA. Panels $\mathbf{A}-\mathbf{C}$ represent the 3 orthogonal planes (A, transverse; $\mathbf{B}$, sagittal; and $\mathbf{C}$, coronal). 2A, Apical 4-chamber view of the fetal heart. 2B, The volume data set is rotated counterclockwise $30^{\circ}$ to $40^{\circ}$ degrees around the $\mathbf{z}$-axis, and the reference dot is moved to the middle of the interventricular septum. $\mathbf{2 C}$, As the volume is rotated clockwise around the $y$-axis, 2 vessels can be visualized leaving the ventricular chambers in parallel. 2D, The reference dot is moved to the vessel leaving the left ventricle; a sagittal orthogonal image shows bifurcation of the pulmonary artery in panel $\mathbf{B}$. DAo indicates descending aorta; IVS, interventricular septum; and PV, pulmonary vein. Other abbreviations are as in Figure 1.

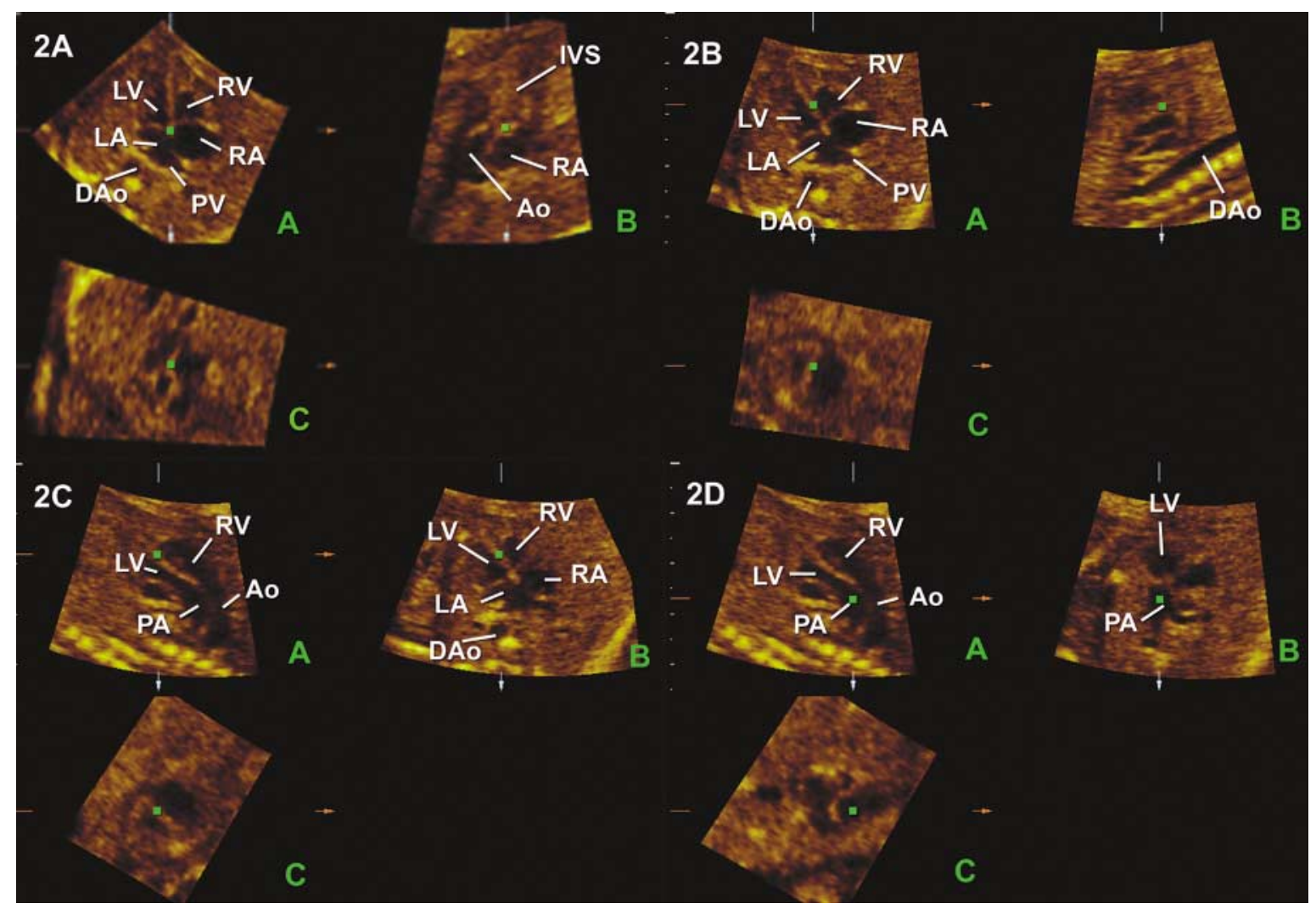


$P<.01)$, (2) a decreased frequency of metabolic acidosis and multiorgan failure (12\% [8 of 68] versus $22 \%$ [56 of 250 ]; $P<.05$ ), (3) shorter hospitalization stays ( $24 \pm 11$ versus $30 \pm 27$ days [mean $\pm \mathrm{SD}] ; P<.01)$, and (4) decreased preoperative mortality ( $0 \%$ [ 0 of 68 ] versus $6 \%$ [ 15 of 250 ]; $P<$ $.05)$ and postoperative mortality ( $0 \%$ [0 of 68$]$ versus $8.5 \%$ [20 of 235]; $P<.05$ ). The definitive treatment for TGA is the "arterial switch" operation..$^{23,24}$ Surgical mortality of the arterial switch operation can be as low as $2 \%$ and is less than $5 \%$ in most hospitals. Preoperative mortality is, therefore, an important issue in the treatment and outcome of fetuses with TGA.22,25,26

This report illustrates that 4-dimensional gray scale and power Doppler STIC can be used to systematically visualize the abnormal relationship of the outflow tracts in fetuses with TGA. Volume acquisition required only clear visualization of the apical 4-chamber view during 2-dimensional ultrasonography for 7.5 to $15 \mathrm{sec}$ onds. The detection rate of TGA by standard obstetric ultrasonographic evaluation is low (Table 1$),{ }^{8-15}$ and this disappointing performance has been attributed to issues related to technical difficulties in consistently imaging the outflow tracts. ${ }^{6,16,18}$

Four-dimensional ultrasonography may overcome technical limitations related to the skills required to obtain appropriate planes of a section. This technology allows for automatic volume acquisition of the fetal heart, including the surrounding areas, while the heart is in motion. Spatiotemporal image correlation stores complete volume data sets of the fetal heart and reduces dependency on the examiner's experience. ${ }^{1-5}$ The user can digitally store the data, optimize and slice the images in an iterative manner as required for diagnosis, and even transport and transmit the images to an expert in

Figure 3. Multiplanar slicing of a normal fetal heart at 20 weeks' gestation. Panels $\mathbf{A}-\mathbf{C}$ represent the 3 orthogonal planes $(\mathbf{A}$, transverse; $\mathbf{B}$, sagittal; and $\mathbf{C}$, coronal). 2A, Apical 4-chamber view of the fetal heart. $\mathbf{2 B}$, The volume data set is rotated counterclockwise $30^{\circ}$ to $40^{\circ}$ around the z-axis, and the reference dot is moved to the middle of the interventricular septum. $\mathbf{2 C}$, As the volume is rotated clockwise around the $y$-axis, 2 vessels can be visualized leaving the ventricular chambers in parallel. 2D, The reference dot is moved to the vessel leaving the left ventricle (aorta); the short axis view of the right ventricular outflow tract is shown in panel $\mathbf{B}$. FO indicates foramen ovale; MB, moderator band; MV, mitral valve; and TV, tricuspid valve. Other abbreviations are as in Figures 1 and 2.

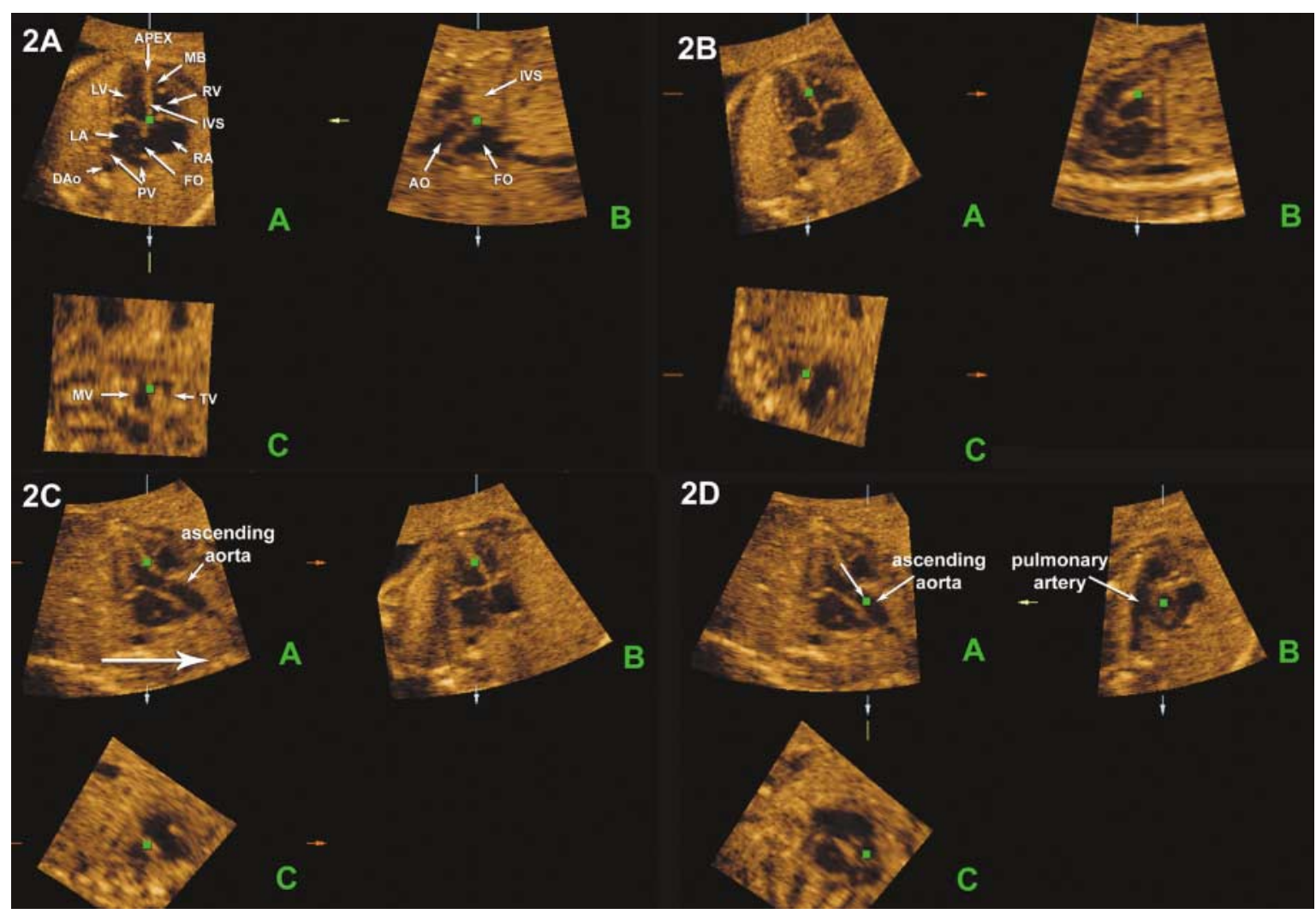




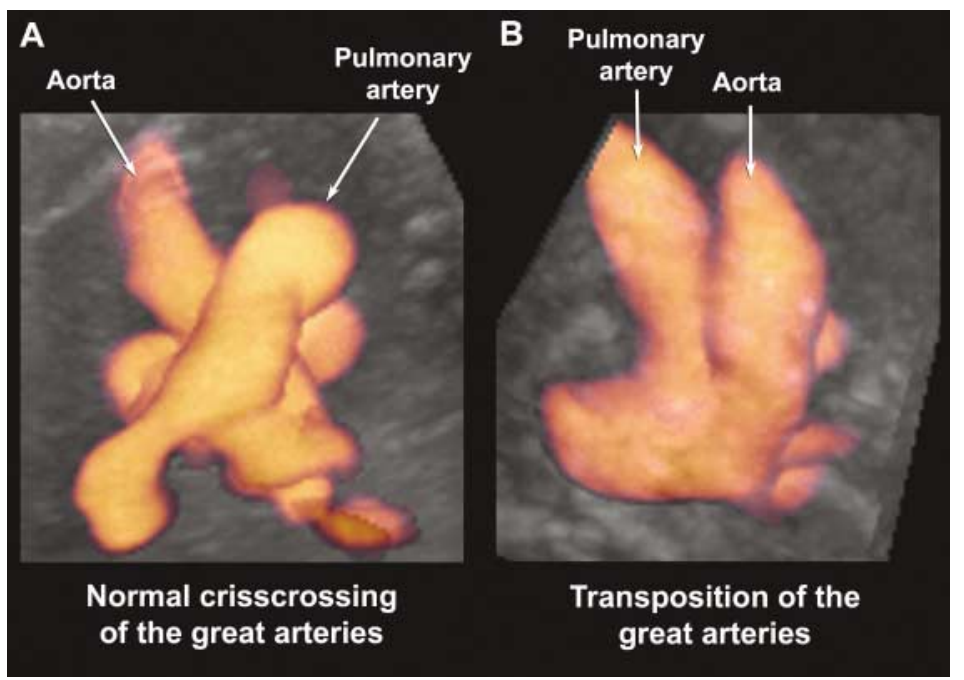

Figure 4. Rendered images from volume data sets of the fetal heart in a normal case ${ }^{5}(\mathbf{A})$ and TGA (B). The volumes were acquired through a transverse sweep of the fetal chest using power Doppler 4-dimensional ultrasonography with STIC. In the normal case, normal crisscrossing of the great arteries is observed, whereas in TGA, the vessels leave the ventricles in parallel. The technique used to render the volume data sets is explained in Figure 5. A, Reproduced from the Journal of Ultrasound in Medicine. ${ }^{5}$

Figure 5. Multiplanar image of the fetal heart during systole acquired by power Doppler STIC. The size and position of the rendering box are shown, as well as the direction of view used to obtain the volume-rendered images of the ventricular outflow tracts in this case of TGA. A, Transverse section. B, Sagittal section. C, Coronal section. D, Volume-rendered image of the outflow tracts. DA indicates ductus arteriosus; and Rt PA, right branch of the pulmonary artery. Other abbreviations are as in Figures 1 and 2.

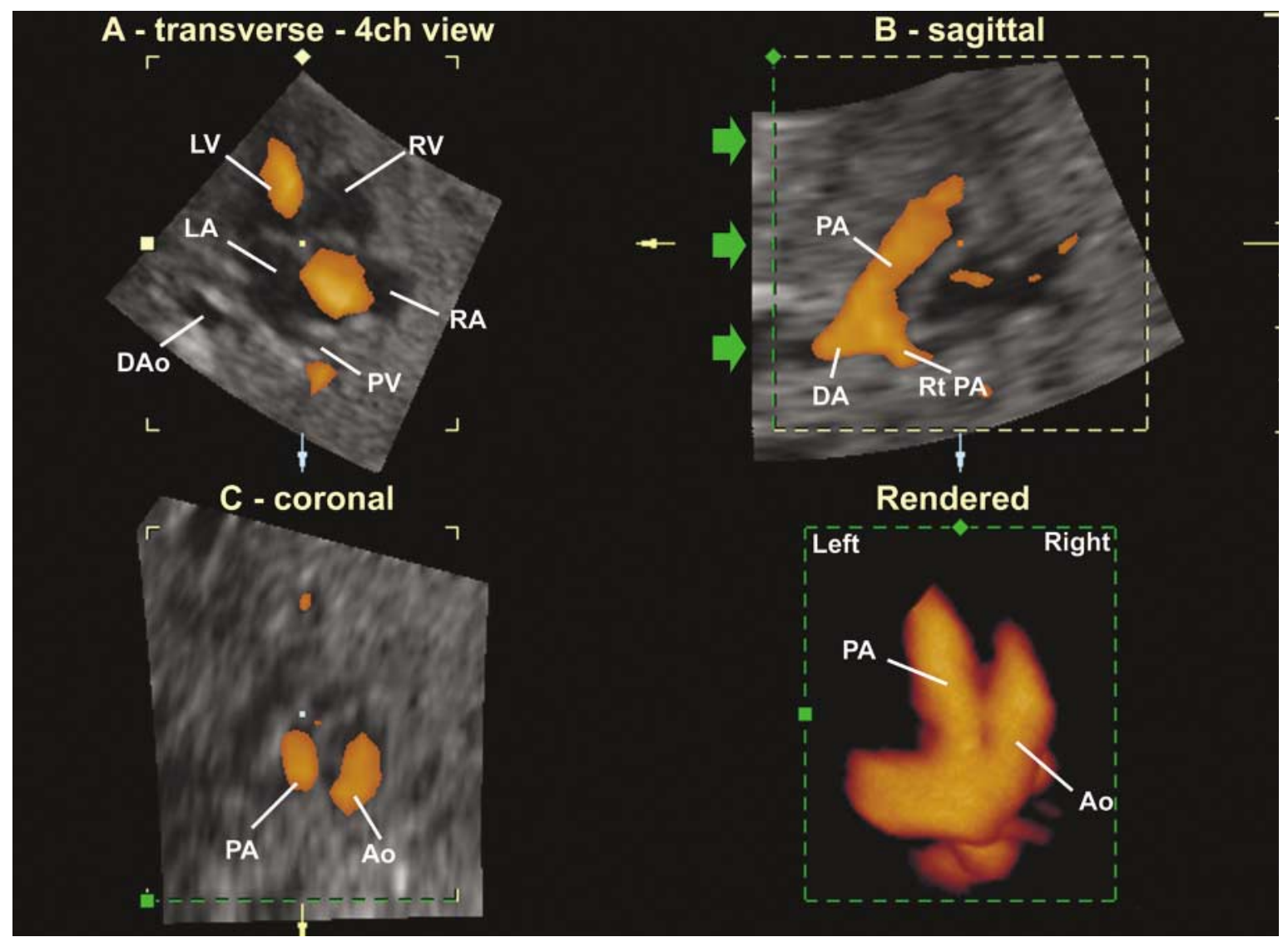


Table 1. Prenatal Detection Rate for TGA

\begin{tabular}{|c|c|c|c|c|c|}
\hline \multirow[b]{2}{*}{ Reference } & \multirow[b]{2}{*}{ Year } & \multirow[b]{2}{*}{ Period } & \multirow[b]{2}{*}{$\mathbf{n}$} & \multicolumn{2}{|c|}{ Detection Rate, \% (n) } \\
\hline & & & & CHD & TGA \\
\hline Stoll et $\left.a\right|^{8}$ & 1993 & 1987-1988 & 26,490 & $9.2(84 / 912)$ & $9.6(6 / 62)$ \\
\hline Stoll et $\mathrm{al}^{9}$ & 1998 & 1990-1993 & 92,021 & $13.7(107 / 779)$ & $51.0(24 / 47)$ \\
\hline Montaña et $a^{10}$ & 1996 & 1990-1994 & 1,589 & $6.1(97 / 1589)$ & $6.3(5 / 80)$ \\
\hline Kirk et $\mathrm{al}^{11}$ & 1997 & 1990-1995 & 16,121 & $65.7(73 / 111)$ & $62.5(5 / 8)$ \\
\hline Bull|12 & 1999 & 1993-1995 & 4,799 & $23.4(1124 / 4799)$ & $3.0(12 / 391)$ \\
\hline Jaeggi et $a^{13}$ & 2001 & 1994-1996 & 240,000 & $15.0(97 / 562)$ & $0.0(0 / 77)$ \\
\hline Garne et al ${ }^{14}$ & 2001 & 1996-1998 & 2,454 & $25.0(613 / 254)$ & $19.0(17 / 91)$ \\
\hline Levi et al ${ }^{15}$ & 2003 & 1990-1993 & 3,633 & $34.0(271 / 798)$ & $24.1(14 / 58)$ \\
\hline
\end{tabular}

CHD indicates congenital heart disease.

fetal cardiology if necessary. ${ }^{2}$ Additional potential clinical benefits of STIC are improved temporal resolution, an increased number of stored 2-dimensional images, and complete rotation of the heart to examine the 3-dimensional multiplanar anatomy around a $360^{\circ}$ axis. ${ }^{1}$ Last, the clear contrast between the images of the normal fetal heart compared with those of the heart affected by TGA as obtained by 4-dimensional rendering techniques (Figure 4, A and B) may result in a better understanding of the congenital cardiac abnormality by the parents and enhance prenatal counseling.

\section{References}

1. DeVore GR, Falkensammer P, Sklansky MS, Platt LD. Spatio-temporal image correlation (STIC): new technology for evaluation of the fetal heart. Ultrasound Obstet Gynecol 2003; 22:380-387.

2. Vinals F, Poblete P, Giuliano A. Spatio-temporal image correlation (STIC): a new tool for the prenatal screening of congenital heart defects. Ultrasound Obstet Gynecol 2003; 22:388-394.

3. Gonçalves LF, Lee W, Chaiworapongsa T, et al. Fourdimensional ultrasonography of the fetal heart with spatiotemporal image correlation. Am J Obstet Gynecol 2003; 189:1792-1802.

4. Gonçalves LF, Lee W, Espinoza J, et al. Four-dimensional fetal echocardiography with spatio-temporal image correlation (STIC): a systematic study of standard cardiac views assessed by different observers [abstract]. Ultrasound Obstet Gynecol 2003; 22(suppl 1):50.
5. Gonçalves LF, Romero R, Espinoza J, et al. Fourdimensional ultrasonography of the fetal heart using color Doppler spatiotemporal image correlation. J Ultrasound Med 2004; 23:473-481.

6. Campbell S, Allan L, Benacerraf B, et al. Isolated major congenital heart disease. Ultrasound Obstet Gynecol 2001; 17:370-379.

7. Bonnet D, Coltri A, Butera G, et al. Detection of transposition of the great arteries in fetuses reduces neonatal morbidity and mortality. Circulation 1999; 99:916-918.

8. Stoll C, Alembik Y, Dott B, Roth PM, De Geeter B. Evaluation of prenatal diagnosis of congenital heart disease. Prenat Diagn 1993; 13:453-461.

9. Stoll $C$, Alembik $Y$, Dott B, et al. Evaluation of prenatal diagnosis of congenital heart disease. Prenat Diagn 1998; 18:801-807.

10. Montaña E, Khoury MJ, Cragan JD, Sharma S, Dhar P, Fyfe D. Trends and outcomes after prenatal diagnosis of congenital cardiac malformations by fetal echocardiography in a well defined birth population, Atlanta, Georgia, 1990-1994. J Am Coll Cardiol 1996; 28:1805-1809.

11. Kirk JS, Comstock CH, Lee W, Smith RS, Riggs TW, Weinhouse E. Sonographic screening to detect fetal cardiac anomalies: a 5-year experience with 111 abnormal cases. Obstet Gynecol 1997; 89:227-232.

12. Bull C. Current and potential impact of fetal diagnosis on prevalence and spectrum of serious congenital heart disease at term in the UK. British Paediatric Cardiac Association. Lancet 1999; 354:1242-1247. 
13. Jaeggi ET, Sholler GF, Jones OD, Cooper SG. Comparative analysis of pattern, management and outcome of pre- versus postnatally diagnosed major congenital heart disease: a population-based study. Ultrasound Obstet Gynecol 2001; 17:380-385.

14. Garne E, Stoll C, Clementi M. Evaluation of prenatal diagnosis of congenital heart diseases by ultrasound: experience from 20 European registries. Ultrasound Obstet Gynecol 2001; 17:386-391.

15. Levi S, Zhang WH, Alexander S, Viart P, Grandjean $\mathrm{H}$. Short-term outcome of isolated and associated congenital heart defects in relation to antenatal ultrasound screening. Ultrasound Obstet Gynecol 2003; 21:532-538.

16. Carvalho JS, Mavrides E, Shinebourne EA, Campbell $S$, Thilaganathan B. Improving the effectiveness of routine prenatal screening for major congenital heart defects. Heart 2002; 88:387-391.

17. Lee W. Performance of the basic fetal cardiac ultrasound examination. J Ultrasound Med 1998; 17 : 601-607.

18. Vinals F, Heredia F, Giuliano A. The role of the three vessels and trachea view (3VT) in the diagnosis of congenital heart defects. Ultrasound Obstet Gynecol 2003; 22:358-367.

19. Abuhamad A. Automated multiplanar imaging: a novel approach to ultrasonography. J Ultrasound Med 2004; 23:573-576.

20. Allan L. Transposition of the great arteries. In: Allan L, Hornberger L, Sharland GK (eds). Textbook of Fetal Cardiology. London, England: Greenwich Medical Media Ltd; 2000:261-273.

21. Allan LD, Sharland GK, Milburn A, et al. Prospective diagnosis of 1006 consecutive cases of congenital heart disease in the fetus. J Am Coll Cardiol 1994; 23:1452-1458.

22. Kumar RK, Newburger JW, Gauvreau K, Kamenir SA, Hornberger LK. Comparison of outcome when hypoplastic left heart syndrome and transposition of the great arteries are diagnosed prenatally versus when diagnosis of these two conditions is made only postnatally. Am J Cardiol 1999; 83:1649-1653.

23. Jatene $A D$, Fontes VF, Paulista PP, et al. Successful anatomic correction of transposition of the great vessels: a preliminary report. Arq Bras Cardiol 1975; 28:461-464.
24. Jatene $A D$, Fontes VF, Paulista PP, et al. Anatomic correction of transposition of the great vessels. J Thorac Cardiovasc Surg 1976; 72:364-370.

25. Maeno $Y$, Kamenir SA, Sinclair B, van der Velde ME, Smallhorn JF, Hornberger LK. Prenatal features of ductus arteriosus constriction and restrictive foramen ovale in d-transposition of the great arteries. Circulation 1999; 99:1209-1214.

26. Soongswang J, Adatia I, Newman C, Smallhorn JF, Williams WG, Freedom RM. Mortality in potential arterial switch candidates with transposition of the great arteries. J Am Coll Cardiol 1998; 32:753-757. 